हींद्यात्क

STOMA

WÖRTERBUCH

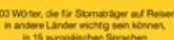

somentents

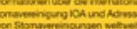

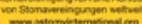

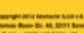

$=$

$+1$
„STOMAWÖRTERBUCH" für reiselustige Stomaträger

Verreisen Stomaträger - also Menschen mit einem künstlichen Ausgang - überhaupt? Und falls ja - reisen sie auch in andere Länder?

Und ob sie das tun. Manches bedarf zwar besonderer Vorbereitungen, aber die meisten Stomaträger lassen sich deswegen nicht vom Reisen ins Ausland abhalten. Unser kleines Wörterbuch erfreut sich daher großer Beliebtheit. Es enthält mittlerweile 103 Worte in 15 verschiedenen europäischen Sprachen, die für Stomaträger auf Reisen wichtig sein können, eine große Hilfe, wenn man am Flughafen oder am Urlaubsort etwas erläutern oder fragen möchte.

Das 16-seitige schmale Heft im DIN langFormat ist Teil der Broschürenreihe der Deutschen ILCO. Es ist unentgeltich - ein Beitrag zu den Versandkosten in Form von Briefmarken wird erbeten - erhältlich bei: Deutsche ILCO e.V., Thomas-Mann-Str. 40, 53111 Bonn Tel. 0228 338894-50, FAX 0228 338894-75, E-Mail:info@ilco.de

\section{Niedergelassene Hämatologen und Onkologen starten neue Webseite}

Der Berufsverband der Niedergelassenen Hämatologen und Onkologen in Deutschland $(\mathrm{BNHO})$ hat seinen Internetauftritt überarbeitet. Die neue Webseite bietet Mitgliedern und Interessierten praktische Serviceleistungen sowie ein umfassendes Informationsangebot.

Die Datenbank enthält die Kontaktdaten der 575 niedergelassenen Hämatologen und Onkologen, die jährlich etwa 600.000 Krebspatienten in Deutschland versorgen.

Für seine Mitglieder hat der BNHO einen passwortgeschützten Bereich eingerichtet. Dort gibt es nützliche Informationen zu Gesetzen und Richtlinien sowie zu relevanten Sachthemen für den Praxisalltag. Quelle: http://www.bnho.de

\title{
Intervallkarzinomen nach Mammographie-Screening
}

Epidemiologisches Krebsregister Nordrhein-Westfalen, Referenzzentrum Mammographie am Universitätsklinikum und Institut für Epidemiologie und Sozialmedizin der Universität Münster berichten über sogenannte Intervallkarzinomraten für NRW. 78 Prozent aller Brustkrebserkrankungen konnten durch Screening-Mammographie diagnostiziert werden.

Bösartige Neubildungen der Brustdrüse stehen bei Frauen in Nordrhein- Westfalen an erster Stelle aller Krebserkrankungen. Jede achte Frau muss im Laufe des Lebens mit der Diagnose Brustkrebs rechnen. Um langfristig die Sterblichkeit an Brustkrebs zu senken, wurde 2005 das Mammographie- Screening-Programm eingeführt: Alle Frauen im Alter zwischen 50 und 69 Jahren werden regelmäßig, das heißt, im Abstand von zwei Jahren, zu dieser qualitätsgesicherten Früherkennungsuntersuchung eingeladen.

Eine Arbeitsgruppe aus Vertretern des Epidemiologischen Krebsregisters Nordrhein-Westfalen, des Referenzzentrums

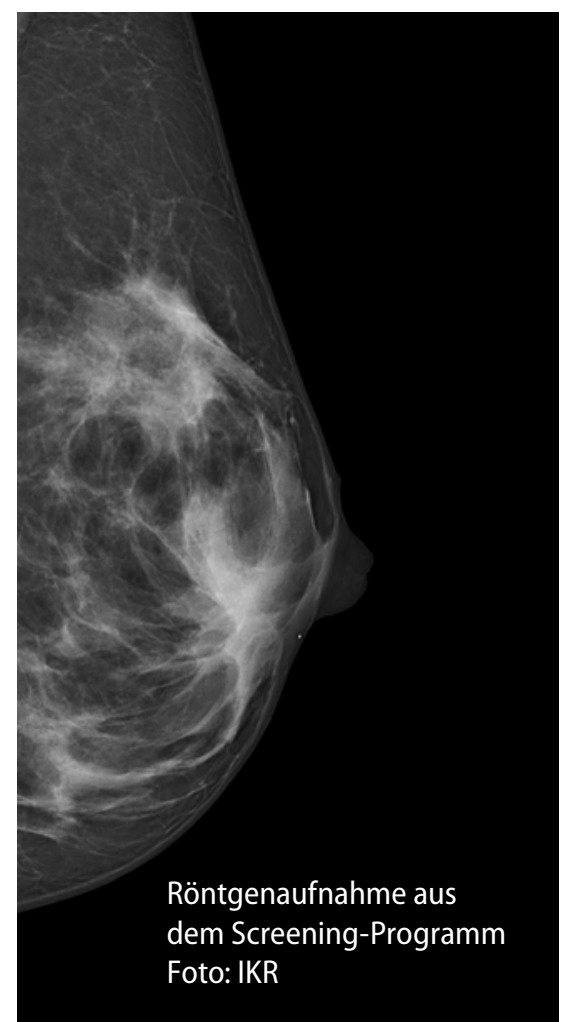

MammographieamUniversitätsklinikum Münster und des Instituts für Epidemiologie und Sozialmedizin der Westfälischen Wilhelms-Universität Münster berichtet nun erstmals über die sogenannten Intervallkarzinomraten für das bevölkerungsreichste deutsche Bundesland Nordrhein-Westfalen. Die wissenschaftliche Auswertung der ersten Untersuchungsrunde von Ende 2005 bis Ende 2008 umfasste über 850.000 ScreeningTeilnehmerinnen und belegt, dass durch die Screening-Mammographie 7.176 Brustkrebserkrankungen erkannt wurden. In den nachfolgenden zwei Jahren traten unter den beim Screening unauffälligen Frauen weitere 2.036 Fälle von neuem Brustkrebs auf. Durch die Screening-Mammographie als alleinige Untersuchung konnten damit 78 Prozent aller Brustkrebserkrankungen in dem Zweijahreszeitraum diagnostiziert werden.

Internationale Publikationen lassen vermuten, dass die im Intervall zwischen zwei Screening-Einladungen diagnostizierten Krebsfälle, die sogenannten Intervallkarzinome, zumeist neu entstanden sind und zum Screening-Zeitpunkt noch nicht diagnostizierbar waren. Die Ergebnisse entsprechen den Vergleichszahlen aus anderen europäischen Ländern und sind ein Beleg dafür, dass in NRW bereits in der Aufbauphase der systematischen Brustkrebs-Früherkennung die Qualität der Programme in anderen europäischen Ländern erreicht wurde. Die Originalarbeit ist Titelthema im Deutschen Ärzteblatt vom 16 . November 2012 (<http://www.aerzteblatt.de/ $\operatorname{archiv} / 132472$ ? $\mathrm{src}=$ toc $>$ ).

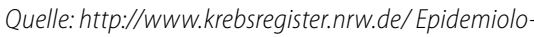
gisches Krebsregister NRW 